\title{
BMJ Open Optimal resection rate for lung cancer in Respiratory Research the UK: how high should we go?
}

\author{
Elizabeth Belcher, ${ }^{1}$ Jenny Mitchell (D) , ${ }^{1}$ Dionisios Stavroulias, \\ Francesco Di Chiara, ${ }^{1}$ Najib Rahman ${ }^{2}$
}

To cite: Belcher E, Mitchell J, Stavroulias D, et al. Optimal resection rate for lung cancer in the UK: how high should we go?. BMJ Open Resp Res 2021;8:e000771. doi:10.1136/ bmjresp-2020-000771

Received 1 October 2020 Accepted 5 July 2021
Check for updates

(c) Author(s) (or their employer(s)) 2021. Re-use permitted under CC BY-NC. No commercial re-use. See rights and permissions. Published by BMJ.

${ }^{1}$ Department of Thoracic Surgery, Oxford University Hospitals NHS Foundation Trust, Oxford, UK ${ }^{2}$ Oxford Respiratory Trials Unit, Oxford University Hospitals NHS Foundation Trust, Oxford, UK

Correspondence to Dr Elizabeth Belcher; elizabeth.belcher@ouh.nhs.uk

\section{ABSTRACT}

Background The optimal resection rate for institutions managing early-stage primary lung cancer is not known. Whether the prognosis of patients who do not proceed to operation is determined by their comorbidities for which they were deemed at prohibitively high-operative risk, or disease progression, is uncertain. We investigated the outcomes of patients with early-stage lung cancer who were considered for surgical management.

Methods We reviewed the outcomes of consecutive patients who were considered for resection of early-stage primary lung cancer at Oxford University Hospitals National Health Service Foundation Trust between 2012 and 2017. Results Between 29 November 2012 and 31 March 2017, 467 consecutive patients underwent resection with curative intent for primary lung cancer (operative group), while 81 patients were deemed resectable but either inoperable or did not wish to proceed to operation (nonoperative group). Reason for not proceeding to resection was cardiovascular in 16 patients $(19.8 \%)$, respiratory in $21(25.9 \%)$, cardiorespiratory in $11(13.6 \%)$, performance status in $8(9.9 \%)$ and patient choice in $25(30.9 \%)$ patients. Sixty-six patients (81.5\%) received an alternative radical treatment. Median follow-up was 169 weeks (IQR 119-246 weeks) in the operative group and 118 weeks (IQR 74-167 weeks) in the non-operative group. Median survival of patients with early-stage lung cancer who did not proceed to operation was 2.5 years; median survival of patients undergoing lung cancer resection was undefined $(p<0.0001)$. Lung cancer was documented as directly or indirectly leading to or contributing to death in 40 patients $(76.9 \%)$. In 11 patients, the cause of death was due to comorbidities $(21.2 \%)$.

Conclusions Patients turned down for operation in a highresection rate UK unit have limited survival due to lung cancer progression. We conclude that 'optimal' resection rates may not have been reached in the UK even in highresection rate centres.

\section{INTRODUCTION}

Surgical resection is the treatment of choice for patients with early-stage lung cancer and higher resection rates are associated with improved long-term survival. ${ }^{1}$ Despite progress within recent years to increase national resection rates, wide variation in rates exists in the $\mathrm{UK}^{23}$ and mortality from lung cancer remains high compared with other developed countries. ${ }^{4}$ It is estimated

\section{Key messages}

What is the fate of the unresected patient with earlystage lung cancer in a high-resection rate thoracic surgery unit?

- Survival of patients with early-stage lung cancer who do not undergo resection is limited; most patients die of lung cancer rather than the comorbidities for which they were considered high risk for surgery, even in a high-resection rate centre.

The pursuit of ever lower operative mortality in lung cancer surgery may limit progress in survival rates of lung cancer in England.

that more deaths could be delayed if all units attained the resection rates of the highest performing centres. ${ }^{1}$ Whether resection rates at these high-performing units are optimal, in terms of the balance of risk between nonsurgical treatment and surgical mortality, remains uncertain.

The ideal resection rate may be defined as a balance between acceptable operative mortality in the surgical cohort and low lung cancer-related mortality in patients deemed inoperable. As a high-resection rate UK thoracic surgical centre with a low 30-day mortality, ${ }^{256}$ we reviewed the outcomes of our non-resected patients with early-stage lung cancer. We examined the hypothesis that should patients turned down for surgery in a high-resection rate centre ultimately succumb to the comorbidities for which they were turned down for surgery, then further efforts to increase resection rates in the highest rate centres are potentially not justified.

\section{METHODS}

A retrospective review was performed on consecutive patients with presumed or proven early-stage primary lung cancer who received a surgical opinion but did not proceed to surgery between 29 November 2012 and 31 March 2017 at Oxford University Hospitals National Health Service Foundation Trust, UK and consecutive patients from a prospective 
database who underwent surgical resection of primary lung cancer. Staging was performed using the Eighth American Joint Committee on Cancer (AJCC) Cancer Staging Handbook. ${ }^{7}$ For the purposes of the study, early stage was defined as stage 0-IIIA. Patients were discussed at multidisciplinary lung cancer meetings and managed according to guidelines on the radical management of patients with lung cancer. $^{89}$

All resectable patients were encouraged to attend a thoracic surgical outpatient appointment for opinion as to operability, as part of our proactive policy of 'surgery as the default option'. Patients were assessed according to guidance for the radical management of lung cancer. ${ }^{89}$ Formal assessment of respiratory function, comorbidities and performance status (PS) was undertaken. Patients with postoperative predicted forced expiratory volume in one second or carbon monoxide transfer factor $<30 \%$ or significant symptoms of breathlessness were assessed by stair climbing with pulse oximetry. A reduction in oxygen saturation of $>4 \%$ was considered prohibitively high risk of resection. ${ }^{10}$ Where patients developed significant symptoms of breathlessness, but oxygen saturation was maintained, consideration was given to enrolling patients within our institutional prehabilitation exercise (SOLACE) programme to improve fitness levels prior to operation. Patients with previous myocardial infarction or symptoms of ischaemia were referred for cardiology opinion and assessed via myocardial perfusion scan. Patients deemed at low risk proceeded to operation; those reported as at intermediate risk of perioperative cardiac events were informed of this risk and proceeded to operation if accepting of this higher-than-average risk. Patients with myocardial perfusion scan reporting high risk of perioperative cardiac event or significant reduction in ventricular function were referred for alternative radical treatments. PS $\geq 2$ was not an absolute contraindication to resection and consideration as to the reason for reduced activity was assessed. Where reduced activity levels were due to long-term neurological or musculoskeletal compromise rather than cardiorespiratory disease, surgical management was considered. Patients considered operable proceeded to resection after informed consent. Following surgery, patients were entered into a surgical CT follow-up programme and were followed by interval imaging for 5 years postoperatively. ${ }^{11}$

Patients who were deemed inoperable due to comorbidities, or who did not wish to proceed to operation, were referred for consideration of alternative treatment. Survival was recorded. Status was confirmed by medical records, electronic patient record and death certification. Cause of death as documented on the medical certificate was examined. Conditions listed in part I of the certificate represent the underlying and direct cause of death and were recorded; diseases listed in part II which contributed to the death but were not part of the direct sequence were noted.

\section{Patient and public involvement}

The development of the research question was informed by the priorities of patients with lung cancer to understand their best treatment options. The results of this study have been used to better inform patients regarding treatment options and improve consent procedure. No specific named patients were involved in this observational study.

\section{Statistical analysis}

We present data as median followed by IQR in parentheses with continuous data and as frequency and percentage for categorical variables. Categorical variables were analysed using $X^{2}$ test. Numerical variables were compared using the Mann-Whitney $\mathrm{U}$ test. The time from date of clinic review or operation to death or last observation was defined as overall survival. Patients were censored at the time they were last known to have been reviewed. Survival curves were calculated using the Kaplan-Meier method. Median survival was estimated and survival differences between groups were evaluated by the log-rank test. Statistical significance was set as a twotailed $p$ value of 0.05 for all tests. All statistical analyses were performed using the GraphPad statistical package (V.9.0 GraphPad Software).

\section{RESULTS}

Between 29 November 2012 and 31 March 2017, 467 consecutive patients underwent resection with curative intent for primary lung cancer (operative group) and 81 patients were deemed resectable but inoperable or did not wish to proceed to operation (non-operative group).

\section{Patient demographics and treatment characteristics of non- operative group}

Characteristics of patients are shown in table 1. Median age of patients in non-operative group was 77.7 years (range 50.8-88.9 years) and 41 patients were men. Pathological confirmation of primary lung cancer was undertaken in 29 patients $(35.8 \%)$. The pathological stage of tumours was according to the eighth edition of the AJCC Cancer Staging Handbook. ${ }^{12}$ Stage was IA1 in 10 patients $(12.3 \%)$, IA2 in $27(33.3 \%)$, IA 3 in $21(25.9 \%)$, IB in $8(9.9 \%)$, IIA in $4(4.9 \%)$, IIB in $8(9.9 \%)$ and IIIA in 3 patients $(3.7 \%)$. Tumours were located in the upper lobes in 45 patients (55.5\%), lower lobes in 29 patients $(35.8 \%)$ and middle lobe in 2 patients $(2.5 \%)$. Five patients $(6.2 \%)$ had tumours in more than one lobe.

In total, 251 comorbidities were identified in 81 patients. Median comorbidity per patient was 3 (range 0-8). Comorbidities were classified into the following groups: cardiac, respiratory, renal, cerebrovascular, peripheral arteriopathy and other. Fifty-four patients 


\begin{tabular}{|c|c|c|c|}
\hline & Operative $(\mathrm{N}=467)$ & Non-operative $(\mathrm{N}=81)$ & \\
\hline & $\mathbf{N}(\%)$ & N (\%) & $P$ value \\
\hline Age (years), median, range & $70.4(18.1-87.7)$ & $77.7(50.8-88.9)$ & $<0.0001$ \\
\hline Gender & & & 0.9042 \\
\hline Female & $234(50.1)$ & $40(49.4)$ & \\
\hline Male & $233(49.9)$ & $41(50.6)$ & \\
\hline Stage & & & 0.0558 \\
\hline 0 & $13(2.8)$ & 0 & \\
\hline $\mathrm{IA} 1$ & $37(7.9)$ & $10(12.3)$ & \\
\hline $\mathrm{IA} 2$ & $119(25.5)$ & $27(33.3)$ & \\
\hline IA3 & $94(20.1)$ & $21(25.9)$ & \\
\hline IB & $44(9.4)$ & $8(9.9)$ & \\
\hline IIA & $21(4.5)$ & $4(4.9)$ & \\
\hline IIB & 79 (16.9) & $8(9.9)$ & \\
\hline IIIA & $60(12.8)$ & $3(3.7)$ & \\
\hline
\end{tabular}

(66.7\%) had comorbidities in two or more groups. Thirty-eight patients had cardiac comorbidities, 42 respiratory, 2 renal, 19 cerebrovascular, 13 peripheral arteriopathy and 65 had other comorbidities. PS was 0 in 9 patients $(11.1 \%), 1$ in 39 patients $(48.1 \%), 2$ in 27 patients $(33.3 \%)$ and 3 in 5 patients $(6.2 \%)$. In one patient, PS could not be determined. Reason for patient not proceeding to resection was cardiovascular in 16 patients $(19.8 \%)$, respiratory in $21(25.9 \%)$, cardiorespiratory in $11(13.6 \%)$, PS in $8(9.9 \%)$ and patient choice in $25(30.9 \%)$ patients.

Sixty-six patients $(81.5 \%)$ received an alternative radical treatment (figure 1). Twenty five (30.8\%) received CT-guided ablative therapy; 23 patients (28.4\%) underwent stereotactic ablative radiotherapy (SABR) and 16 patients $(19.8 \%)$ underwent radical radiotherapy. Two patients $(2.5 \%)$ underwent radical treatment for synchronous primary tumours. One patient received SABR and radical radiotherapy and one patient received SABR and ablation. Fifteen patients $(18.5 \%)$ received no alternative radical treatment (figure 1 ).

\section{Patient demographics and treatment characteristics of operative group}

Surgery with curative intent was performed on 467 consecutive patients with primary lung cancer (table 1). Median age was 70.4 years (18.1-87.7 years) and 233 patients $(49.9 \%)$ were men. Patients who did not proceed to operation were significantly older than patients who underwent resection $(p<0.0001)$. There was no difference in ethnicity between the operative and non-operative groups ( $97 \%$ vs $98.5 \%$ white patients, respectively $\mathrm{p}=0.48$ ). Operations were performed by one of six thoracic surgeons.

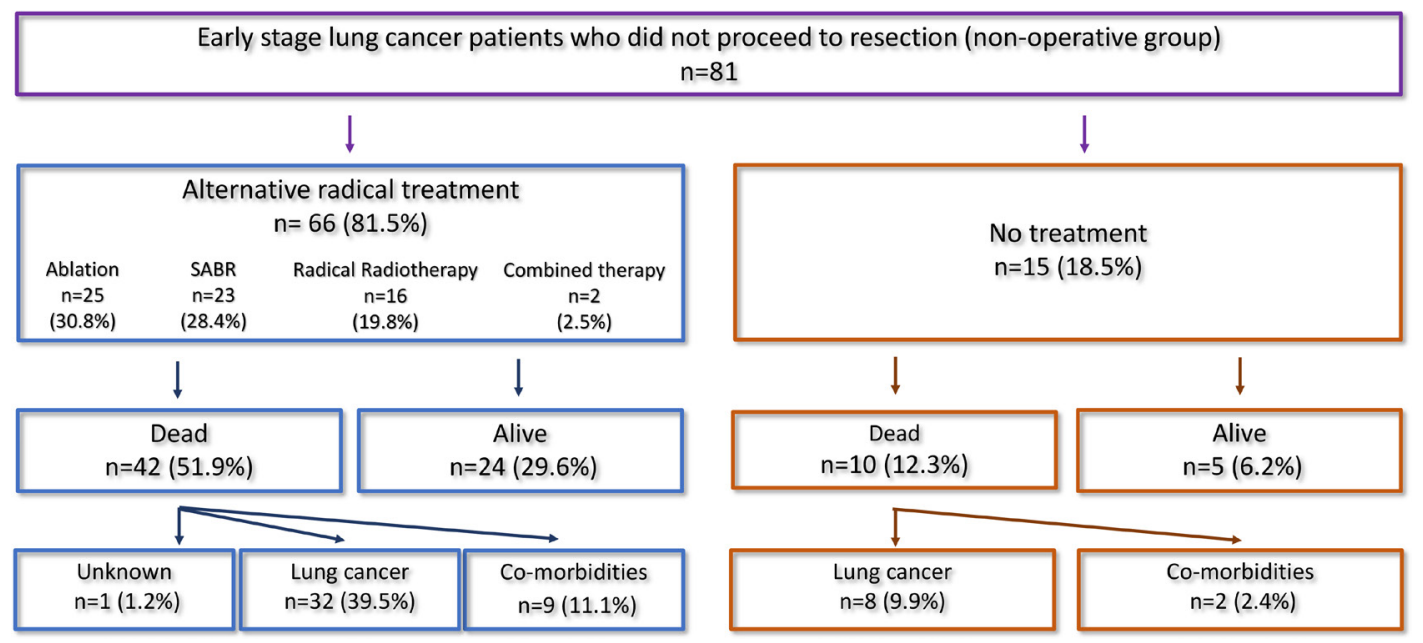

Figure 1 Treatment pathways and outcomes of non-operative group of patients with early-stage primary lung cancer. SABR, stereotactic ablative radiotherapy. 
Of the 467 patients who underwent resection, 298 $(63.8 \%)$ were performed via video-assisted thoracoscopic surgery and $169(36.2 \%)$ at thoracotomy. The histological type of lung cancer was adenocarcinoma in 288 patients $(61.7 \%)$, squamous cell carcinoma in 105 patients $(22.5 \%)$, neuroendocrine in 53 patients $(11.3 \%)$ and other in 21 patients $(4.5 \%)$. The pathological stage of tumours was according to the eighth edition of the AJCC Cancer Staging Handbook. ${ }^{12}$ Stage was 0 in 13 patients $(2.8 \%)$, IA in $250(53.5 \%)$, IB in $44(9.4 \%)$, IIA in $21(4.5 \%)$, IIB in $79(16.9 \%)$ and IIIA in $60(12.8 \%)$. There was no difference in stage between the operative and non-operative group $(\mathrm{p}=0.056$, table 1$)$. Surgical procedures were pneumonectomy in 11 patients $(2.4 \%)$, extended resection in 13 patients $(2.8 \%)$, bilobectomy in 17 patients $(3.6 \%)$, lobectomy in 362 patients $(77.5 \%)$ and sublobar resection in 64 patients $(13.7 \%)$.

\section{Outcomes}

Median follow-up was 169 weeks (IQR 119-246 weeks) in the operative group and 118 weeks (IQR 74-167 weeks) in the non-operative group. Operative 30-day mortality was $1.07 \%$ ( 5 of 467 died within 30 days of operation). Cause of death was pneumonia and sepsis leading to multiorgan failure in three patients, cerebral infarction in one patient and ischaemic bowel and multiorgan failure in one patient. Median survival of patients undergoing lung cancer resection was not reached in the follow-up period; median survival of non-operative patients who did and did not receive alternative treatment was 2.5 years and 1.8 years, respectively $(\mathrm{p}<0.0001)$ (figure 2$)$. Fifty-two of the 81 patients in the non-operative group are known to have died to date. Cause of death was available in 51 of 52 patients $(98.0 \%)$. In 11 patients, the cause of death was due to comorbidities (21.2\%). Lung cancer was documented as the direct cause or contributing factor to death in 40 patients $(76.9 \%)$; in 34 patients $(65.4 \%)$ lung cancer was the direct cause of death (figure 3 ).

Of the 11 patients who died of their comorbidities, 9 patients $(81.8 \%)$ received alternative radical treatment;

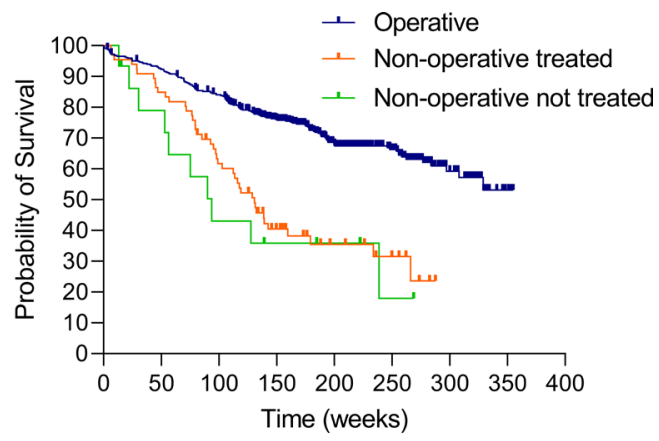

Number at Risk

$\begin{array}{lllll}\text { Operative } & 467 & 387 & 177 & 36 \\ \text { Non-operative treated } & 66 & 40 & 12 & 1 \\ \text { Non-operative not treated } & 15 & 7 & 4 & 1\end{array}$

Figure 2 Survival of operative and non-operative groups of patients with early-stage lung cancer.

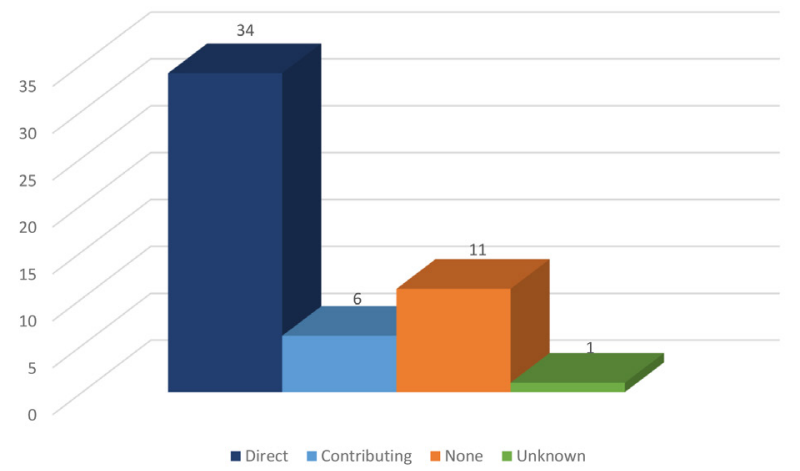

Figure 3 Lung cancer as cause of death in the nonoperative group $(n=52)$.

6 patients (54.5\%) received SABR, 3 patients (27.3\%) underwent CT-guided ablative therapy and 2 patients (18.2\%) received no radical treatment. Of the 40 patients in whom lung cancer was documented as directly or indirectly leading to or contributing to death, 32 patients $(80 \%)$ received alternative radical treatment; 13 patients $(32.5 \%)$ received radical radiotherapy, 11 patients $(27.5 \%)$ received SABR, 7 patients $(17.5 \%)$ underwent CT-guided ablative therapy, $1(2.5 \%)$ patient underwent radical radiotherapy and SABR, and 8 patients $(20.0 \%)$ received no radical treatment. There was no difference in survival between patients declining surgery and receiving SABR and those evaluated as functionally not operable (median survival 215 weeks vs 116 weeks, respectively $\mathrm{p}>0.1$ ). Median survival of patients undergoing lung cancer resection was not defined in the follow-up period; median survival of patients declining surgery was 238.7 weeks $(\mathrm{p}<0.05)$ (figure 4$)$.

\section{DISCUSSION}

We have reviewed outcomes in patients with lung cancer who did not proceed to resection in a high-resection rate thoracic surgery unit in the UK. The fate of non-operative patients is generally unknown but, in this study where we have taken the most conservative measures, death was directly due to lung cancer progression in $65.4 \%$ of patients, rather than the comorbidities for which they were turned down for surgery. Given that our resection rate is associated with low operative 30-day mortality and that outcomes for patients who do not proceed to surgery are poor, we postulate that our resection rate may be suboptimal and that more lives may be saved if higher risk patients were offered surgery. The pursuit of ever lower operative mortality in lung cancer surgery may have limited progress in survival rates of lung cancer and even centres with high-resection rates may be able to increase their rates further. This study supports the hypothesis that resection rates may be suboptimal even in higher resection rate UK centres.

Increasing the resection rate in fit, early-stage patients should be a priority for quality improvement in lung 


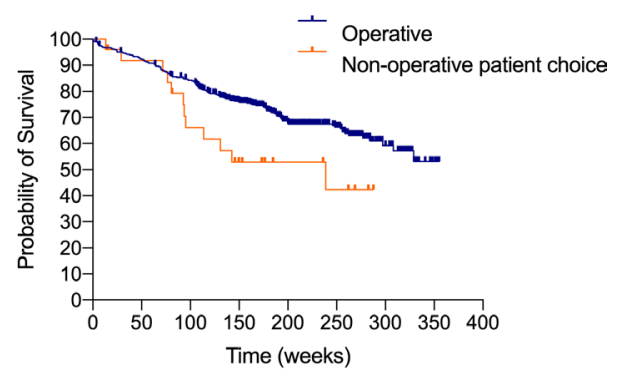

Number at Risk

$\begin{array}{lllll}\text { Operative } & 467 & 387 & 177 & 36 \\ \text { Non-operative patient choice } & 25 & 16 & 7 & 1\end{array}$

Figure 4 Survival of operative patients and non-operative patients declining lung cancer surgery.

cancer surgery. ${ }^{2}$ Despite year-on-year increases in resection rate in the UK, variation exists between units with up to twofold differences in radical treatment rates which remain unexplained. ${ }^{2}{ }^{13}$ It has been estimated that a further 1000 patients per year would receive surgery if the variation was addressed. ${ }^{2}$ Units who seek to extend their criteria of operability are understandably concerned about the risk of higher operative mortality; however, an increase in resection rate is not necessarily associated with adverse early outcomes. In a study from Finland, an increase in resection rate over time, mainly due to inclusion of higher risk patients, resulted in fewer complications and shorter hospital stays while increasing recurrence-free 5-year survival from $64.0 \%$ to $76.8 \% .^{14}$ Goldsmith et $a l^{15}$ demonstrated that introduction of a prehabilitation programme to a surgical unit was associated with improvement in dyspnoea scores, PS, level of activity and frailty, particularly in high-risk patients. Prehabilitation facilitated resection in previously inoperable patients without increases in complications, length of hospital stay or mortality. ${ }^{15}$

A National Lung Cancer Audit Spotlight report on curative intent treatment of stage I-IIIa non-small cell lung cancer (NSCLC) reported comorbidities as being a reason for not having surgery in only $11 \%$ of patients; however, data on comorbidities were missing in approximately $75 \%$ of patients. ${ }^{13}$ Information regarding comorbidities, survival and cause of death in patients who do not receive surgery is vital to assess optimal resection rates and to appropriately inform patients during the consent process. In the present study, cardiovascular comorbidities either in isolation or together with respiratory comorbidity accounted for $33.3 \%$ of contraindications for surgery. Cardiovascular disease is of particular concern in patients considered for surgical resection. ${ }^{16}$ Using patients within the Public Health England Cancer Registry with stage I-IIIA lung cancer, modelling suggested that increasing resection in patients with cardiovascular comorbidity to the levels of the highest resecting region would increase 1-year survival. ${ }^{17}$ Whether further increases in these high-resection rates units would further increase survival is not known.
An ideal resection rate may be defined as a balance between acceptable operative mortality in the surgical group and low lung cancer-related mortality in those patients deemed inoperable. In order to understand optimal resection rates, equal focus on outcomes of patients with non-operative lung cancer as well as operative mortality in surgical patients is required-historically, this has not been the case with the large focus being on operative mortality only. ${ }^{13}$ In the present study, patient choice accounted for $31 \%$ of patients who did not proceed to resection. For those patients who are uncertain regarding surgery, it is vital that in addition to perioperative risk, long-term outcomes of surgical and non-surgical management are used to inform individuals, and this may in turn encourage higher resection rates. 'Framing effect' is a well-recognised cognitive bias where people decide on options based on whether the options are presented with positive or negative outcomes. ${ }^{18}$ The conduct of the consent process by the thoracic surgeon is a crucial factor in determining patient willingness to undergo surgery. Attractiveness of surgery versus alternative treatments has been shown to be higher when patients receive information in terms of life expectancy and probability of living. ${ }^{19}$ Patients should be consented appropriately with balance given to operative and non-operative survival outcomes.

The present study has a number of limitations. The study is subject to the attendant biases of retrospective analyses. It is possible that death certification overestimated lung cancer as the cause of death in the nonoperative group. Medical practitioners are required to complete a medical certificate of cause of death (MCCD) to the best of their knowledge and belief. Conditions listed in part I of the MCCD represent the underlying and direct cause of death. In part II of the certificate, diseases which contributed to the death but were not part of the direct sequence are listed. These conditions must be known and suspected to have contributed to the death, not merely be other conditions which were present at the time of death..$^{20}$ In a study investigating the impact of cause-of-death misclassification on the estimated incidence of lung cancer deaths, misclassification resulted in substantial underestimation of the true cumulative incidence of lung cancer deaths in NSCLC, rather than overestimation. ${ }^{21}$

It is not clear that those patients in whom operative treatment was not considered would have survived after thoracic surgery, but it is clear that lung malignancy predominates as the direct cause of death (rather than the comorbidities) in this patient group. The nonoperative group are by definition heterogeneous in that a proportion of patients $(31 \%)$ refused surgery, whereas others were considered too high risk to operate driven by comorbidities in the setting of a national average operative mortality of $1 \%-2 \%{ }^{2}$ Inclusion of the 'refusing' patients will act to dilute any signal favouring surgical treatment in this analysis, as they are less likely to have limiting comorbidities, given that they were offered surgery. 
In conclusion, although higher surgical resection rates are associated with better outcomes, the 'optimal' (acceptable operative risk, most favourable outcome from cancer and its treatment) resection rate remains to be determined. Our data suggest that even in the highest resection rate centres, we may not be aggressive enough in pursuing surgery. Further prospective research focused on the highest risk patients is now required to ensure optimal treatment is offered, and we would encourage all centres to carefully document the outcomes of patients who are considered 'unfit' for surgery in order to provide balance in decision-making for future patients.

Contributors EB contributed to the study design, literature search, data collection, data analysis, data interpretation and writing. JM contributed to the study design, literature search, data collection, data analysis, data interpretation and writing. DS and FDC contributed to the study design, drafting the article and critical revision of the article. NR contributed to the study design, data analysis and interpretation, and writing. All authors approved the final version of the article.

Funding The authors have not declared a specific grant for this research from any funding agency in the public, commercial or not-for-profit sectors.

Competing interests None declared.

Patient and public involvement Patients and/or the public were not involved in the design, or conduct, or reporting, or dissemination plans of this research.

Patient consent for publication Not required.

Ethics approval As this is an evaluation of our standard of care, ethical approval was not required in our institution, and the study was approved by the audit office of the Oxford University Hospitals NHS Foundation Trust.

Provenance and peer review Not commissioned; externally peer reviewed.

Data availability statement Data are available upon reasonable request. Anonymised data on the patient dataset used in this study are stored according to NHS data storage guidelines. If you would like to access these data, please contact the corresponding author.

Open access This is an open access article distributed in accordance with the Creative Commons Attribution Non Commercial (CC BY-NC 4.0) license, which permits others to distribute, remix, adapt, build upon this work non-commercially, and license their derivative works on different terms, provided the original work is properly cited, appropriate credit is given, any changes made indicated, and the use is non-commercial. See: http://creativecommons.org/licenses/by-nc/4.0/.

ORCID iD

Jenny Mitchell http://orcid.org/0000-0001-5644-475X

\section{REFERENCES}

1 Riaz SP, Lüchtenborg M, Jack RH, et al. Variation in surgical resection for lung cancer in relation to survival: population-based study in England 2004-2006. Eur J Cancer 2012;48:54-60.
2 Royal College of Physicians. National lung cancer audit. lung cancer clinical outcomes publication 2019 (for the 2017 audit period). London: RCP, 2020.

3 West of Scotland Cancer Network. Audit report: lung quality performance indicators. clinical audit data: 01 January 2018 to 31 December 2018. WoSCAN, 2020.

4 Arnold M, Rutherford MJ, Bardot A, et al. Progress in cancer survival, mortality, and incidence in seven high-income countries 1995-2014 (ICBP SURVMARK-2): a population-based study. Lancet Oncol 2019;20:1493-505.

5 Royal College of Physicians. National lung cancer audit annual report 2017 (for the audit period 2016). London: RCP, 2018.

6 Royal College of Physicians. National lung cancer audit annual report (for the audit period 2018) version 2. London: Royal College of Physicians, 2021.

7 Rami-Porta R, ed. IASLC Staging Manual in Thoracic Oncology. North Fort Myers, FL: Editorial Rx Press, 2016.

$8 \mathrm{Lim} \mathrm{E}$, Baldwin D, Beckles M, et al. Guidelines on the radical management of patients with lung cancer. Thorax 2010;65:iii1-27.

9 Brunelli A, Charloux A, Bolliger CT, et al. ERS/ESTS clinical guidelines on fitness for radical therapy in lung cancer patients (surgery and chemo-radiotherapy). Eur Respir J 2009;34:17-41.

10 Brunelli $\mathrm{A}$, Refai $\mathrm{M}$, Xiumé $\mathrm{F}$, et al. Oxygen desaturation during maximal stair-climbing test and postoperative complications after major lung resections. Eur J Cardiothorac Surg 2008;33:77-82.

11 Mitchell J, Benamore R, Gleeson F, et al. Computed tomography follow-up identifies radically treatable new primaries after resection for lung cancer. European Journal of Cardio-Thoracic Surgery 2019;145:4771-8.

12 Edge S, Byrd DR, Compton CC, et al, eds. AJCC Cancer Staging Handbook. New York: Springer, 2010.

13 Royal College of Physicians. Spotlight report on curative-intent treatment of stage 1-111a non-small-cell lung cancer, July 2020. London: RCP, 2020.

14 Helminen O, Valo J, Andersen $\mathrm{H}$, et al. Real-World guideline-based treatment of lung cancer improves short- and long-term outcomes and resection rate: a population-based study. Lung Cancer 2020;140:1-7.

15 Goldsmith I, Chesterfield-Thomas G, Toghill H. Pre-Treatment optimization with pulmonary rehabilitation in lung cancer: making the inoperable patients operable. EClinicalMedicine 2021;31:100663.

16 Brunelli A, Cassivi SD, Fibla J, et al. External validation of the recalibrated thoracic revised cardiac risk index for predicting the risk of major cardiac complications after lung resection. Ann Thorac Surg 2011;92:445-8

17 Welch CA, Sweeting MJ, Lambert PC, et al. Impact on survival of modelling increased surgical resection rates in patients with nonsmall-cell lung cancer and cardiovascular comorbidities: a VICORI study. Br J Cancer 2020;123:471-9.

18 Kahneman D, Tversky A. Prospect theory: an analysis of decision under risk. Econometrica 1979;47:263-91.

19 McNeil BJ, Pauker SG, Sox HC, et al. On the elicitation of preferences for alternative therapies. $N$ Engl J Med 1982;306:1259-62

20 HM Government. Completing a medical certificate of cause of death (MCCD). Available: https://www.gov.uk/government/publications/ guidance-notes-for-completing-a-medical-certificate-of-cause-ofdeath [Accessed 3 Mar 2021]

21 Tan KS. Misclassification of the actual causes of death and its impact on analysis: a case study in non-small cell lung cancer. Lung Cancer 2019;134:16-24. 